$\mathbb{T}$ periodica polytechnica

Civil Engineering

$55 / 1(2011) 81+86$

doi: 10.3311/pp.ci.2011-1.10

web: http://www.pp.bme.hu/ci

(c) Periodica Polytechnica 2011

RESEARCH ARTICLE

\section{Multiple constrained sizing-shaping truss-optimization using ANGEL method}

\author{
Anikó Csébfalvi
}

Received 2010-10-15, revised 2011-01-25, accepted 2011-02-11

\begin{abstract}
The aim of this study to demonstrate that the previously developed ANGEL algorithm can be efficiently used for multiple constrained sizing-shaping truss optimization problems. The applied hybrid method ANGEL, which was originally developed for simple truss optimization problems combines ant colony optimization (ACO), genetic algorithm (GA), and local search strategy (LS). ACO and GA search alternately and cooperatively in the solution space. In ANGEL, the traditional stochastic mutation operator is replaced by the local search procedure as a deterministic counterpart of the stochastic mutation. The feasibility is measured by the maximal load intensity factor computed by a third order path-following method. The powerful LS algorithm, which is based on the local linearization of the set of the constraints and the objective function, is applied to yield a better feasible or less unfeasible solution when ACO or GA obtains a solution. In order to demonstrate the efficiency of ANGEL in the given application area, a well-known example is presented under multiple constraints.
\end{abstract}

\section{Keywords}

ANGEL hybrid heuristic method; shaping-sizing truss optimization

\section{Anikó Csébfalvi}

Department of Structural Engineering, University of Pécs, Boszorkány út 2, H7624 Pécs, Hungary

e-mail: csebfalv@witch.pmmf.hu

\section{Introduction}

The traditional meaning of the truss optimization is a minimal mass design in terms of the cross-sectional areas as design variables while fixed geometry is supposed [1,2]. However, the total mass of the truss highly depends upon the join positions. The early works for simultaneous sizing-shaping truss optimization have been appeared about the seventies of the last century [3-8] where the solutions are obtained by successive iteration using gradient-based methods, although, such an optimization problem is nonconvex and nonlinear. Therefore, the solution of the implicit equilibrium equation systems and the computation of the gradients for the traditional algorithms required a prescribed move-limit for the cross-sectional and geometry design variables. Important to note, that the success of the traditional method highly depends on the initial design. However, if a good jumping-off point is found in the local environment of the optimal solution, the linearized gradient-based methods seem very accurate.

The aim of this study to demonstrate that the previously developed ANGEL algorithm [9, 12] can be efficiently used for multiple constrained sizing-shaping truss optimization problems.

The session 2 contains the general formulation of the optimization problems and the basic formulas of the computation of structural constraints, where stress, displacement, and buckling constraints are considered. The equilibrium equation systems of the geometrically linear and nonlinear structural model are based on the stationary theory of the total potential energy function as well.

In session 3, the hybrid meta-heuristic method is discussed, which is an extended version of the previously published method applied for discrete and continuous optimization problems. The naming ANGEL of the proposed method is an acronym, combines ant colony optimization (ACO), genetic algorithm (GA), and local search strategy (LS). In session 4, the linearized formulas of applied local search strategy is presented.

In session 5, throughout the Pedersen's benchmark example [6] the efficiency of the proposed method is demonstrated where four different constrained cases are considered. With the help of the statistical evaluation of the best feasible solutions of thirty 
independent runs, has been proven the stability and dependability of the process.

\section{The optimization problem}

In this paper, the single-objective continuous sizing-shaping optimization problem is considered as minimal weight design subjected to equilibrium of state variables, nodal displacements, and stress constraints.

$$
\begin{aligned}
& W(X, Y) \rightarrow \min , \\
& \{X, Y\} \in \mathbf{\Theta} \subseteq \mathbf{\Omega},
\end{aligned}
$$

where the design space is $\boldsymbol{\Omega}$ and the subspace of the feasible designs is denoted by $\Theta$ :

$$
\begin{aligned}
& \boldsymbol{\Omega}=\left\{\{X, Y\} \mid X_{i}, \in\left[X^{L}, X^{U}\right], Y_{j}, \in\left[Y^{L}, Y^{U}\right]\right\} \text { (3) } \\
& \boldsymbol{\Theta}=\left\{\{X, Y\} \mid\{X, Y\} \in \mathbf{\Omega}, G_{k}(X, Y) \in\left[G^{L}, G^{U}\right]\right\}(4)
\end{aligned}
$$

where $G_{k}, k \in\{1,2, \ldots, C\}$ are the implicit structural response constraints of the structure, $X=\left(X_{1}, X_{2}, \ldots, X_{N}\right)$, $i \in\{1,2, \ldots, M\}$ is the vector of the continuous sizing variables, $Y=\left(Y_{1}, Y_{2}, \ldots, Y_{M}\right), j \in\{1,2, \ldots, N\}$ is the vector of the continuous shift variables.

In this paper, a design is represented by the set of $\{W, \lambda, X, Y, \Phi\}$, where $W$ is the weight of the structure, $\lambda=$ $\lambda(X, Y),(0 \leq \lambda \geq 1)$ is the maximal load intensity factor, $\{X, Y\}$ is the current set of the cross-sections of member groups, and $\Phi=\Phi(X, Y)$ is the current fitness function value.

The structural response analysis is based on the geometrically nonlinear total potential energy function of elastic system, which can be described in terms of the vector of sizing and shifting design variables.

The structural state variables are the load intensity factor and the vector of nodal displacements.

$$
V(X, Y, D, \lambda)=U(X, Y, D, \lambda)-D^{T} \lambda F
$$

where $U(X, Y, D, \lambda)$, the nonlinear strain energy function, is depend on the design variables and the state variables. The vector of nodal displacements and the external loads are denoted by $D \in \mathfrak{R}^{D O F}$, and $F \in \mathfrak{R}^{D O F}$, where $D O F$ is the number of degrees of freedom.

According to the stationary theory of the total potential energy function, the equilibrium equation system of state variables is obtained by differentiation of energy function (5).

$$
\frac{\partial V(X, Y,, D, \lambda)}{\partial D}=\frac{\partial U(X, Y,, D, \lambda)}{\partial D}-\lambda F=0 .
$$

In order to measure the feasibility of the current design (e.g. measure of the satisfaction of the constraints), we have to solve the equilibrium equation system (6) in terms of the state variables. In this study, the response functions and the maximal load intensity factor $\lambda$ is computed by a higher order nonlinear path-following method [13]. In order to measure the goodness of the solutions ANGEL uses a feasibility-oriented fitness function $(\Phi)$ which is based on a set of criteria introduced previously by Deb [14]:

(1) Any feasible solution is preferred to any infeasible solution,

(2) Between two feasible solutions, the one having a smaller weight is preferred,

(4) Between two infeasible solutions, the one having a larger load intensity factor is preferred.

It should be stressed that our fitness function exploits the fact, that the maximal load intensity factor $\lambda$ given by the applied path-following method is a "natural" measure of feasibility.

During the optimization process, each phases of the proposed ANGEL hybrid metaheuristic method are governed by the following fitness function $\Phi=\Phi(X, Y) \quad(0 \leq \Phi \leq 2)$ :

$$
\Phi=\left\{\begin{array}{ccc}
2-\frac{W-W^{L}}{W^{U}-W^{L}} & & \lambda=1 \\
\lambda & \text { if } & \\
\lambda & & \lambda<1
\end{array},\right.
$$

where $W^{L}\left(W^{U}\right)$ is a lower (upper) bound of the weight in the given design space: $W^{L}=\min \{W(X, Y) \mid\{X, Y\} \in \mathbf{\Omega}\}$, $W^{U}=\max \{W(X, Y) \mid\{X, Y\} \in \mathbf{\Omega}\}$. In the proposed approach, it is assumed that the heaviest (lightest) design is feasible (infeasible).

\section{The ANGEL method}

In the presented ANGEL algorithm (Fig. 1), the traditional mutation operator is replaced by the local search procedure as a deterministic counterpart of the stochastic mutation. That is, rather than introducing small random perturbations into the offspring solution, a gradient based deterministic local search is applied to improve the solution until a local optimum is reached. In other words, random perturbation is replaced by the "best" perturbation.

The main procedure of the proposed hybrid metaheuristic follows the repetition of these two steps:

(1) ACO with LS and

(2) GA with LS.

The hybrid algorithm is based on three operators: random selection $(\mathrm{ACO}+\mathrm{GA})$, random perturbation $(\mathrm{ACO})$, and random combination (GA).

The initial population of the process is a totally random set. The random perturbation and random combination operators based on normal distribution - use a tournament selection operator, to select a "more or less good" solution from the current population using the well-known discrete inverse method. The procedures use a uniform random number generator in the inverse method. We have to mention, that in our algorithm in the GA phase, an offspring not necessarily will be the member of the current population, and a parent not necessarily will die after mating. The reason is straightforward because of our algorithm 
uses a very simple rule: If the current design is better than the worst solution of the current population, than the better one will replace the worst solution.

The Fig. 1 contains three phases: (i) RANDOM POPULATION, (ii) ANT COLONY OPTIMIZATION, and (iii) GENETIC ALGORITHM, where $Z$ signify the common vector of continuous sizing and shift design variables.

It should be noted, that the main framework of ANGEL is very similar to another hybrid algorithm [15] according to the same goal and common roots and basic features.

\section{The local search procedure}

In the presented hybrid method, a gradient based, deterministic local search (LS) is implemented to improve the solution until a local optimum or the maximal number of iterations is reached. The LS procedure is based on two linear programming (LP) models and calls a LP solver to solve them.

When the current solution of the iterative process is feasible then LS tries to find a better solution in its local neighbourhood without violating constraints:

$$
\begin{gathered}
\Delta W(\Delta Z) \rightarrow \min , \\
G_{j}(Z)+\sum_{i=1}^{N} \frac{\partial G_{j}(Z)}{\partial Z_{i}} * \Delta Z_{i} \in\left[G_{j}^{L}, G_{j}^{U}\right], \\
j \in\{1,2, \ldots, M\} \\
\Delta Z_{i} \in\left[\Delta Z_{i}^{L}, \Delta Z_{i}^{U}\right], \quad i \in\{1,2, \ldots, N\}
\end{gathered}
$$

When the current solution of the iterative process is infeasible then LS tries to find a better (feasible or less infeasible) solution in its local neighbourhood:

$$
\sum_{j=1}^{M}\left(\Delta G_{j}^{L}+\Delta G_{j}^{U}\right) \rightarrow \min
$$

$$
\begin{aligned}
& G_{j}(Z)+\sum_{i=1}^{N} \frac{\partial G_{i}(Z)}{\partial Z_{i}} * \Delta Z_{i} \in\left[G_{j}^{L}-\Delta G_{j}^{L}, G_{j}^{U}+\Delta G_{j}^{U}\right], \\
& j \in\{1,2, \ldots, M\}
\end{aligned}
$$

$$
\Delta Z_{i} \in\left[\Delta Z_{i}^{L}, \Delta Z_{i}^{U}\right], \quad i \in\{1,2, \ldots, N\}
$$

$$
\Delta W(\Delta Z) \leq \varepsilon
$$

\section{Numerical example}

The presented ANGEL method has been applied for a wellknown bridge problem of the simultaneous sizing-shaping optimization. Pauli Pedersen [6] has introduced this example first time. He proposed a parabolic shape for initial layout, which is displayed on Fig. 2. The objective function is the weight of the structure subjected to stress, displacements, and stability constraints. According to the moving loads acting on the bottom joints, five load cases are considered simultaneously. The sizing variables are grouped into 13 group variables because of the structural symmetry (Tab. 11. The shift variables are the horizontal and vertical positions of the joints 5, 6, 7, 12, and 11 . The initial data of the applied material properties and structural constraints are adopted from the literature (Tab. 2).

In this study, stainless steel tubular cross sections are considered as design variables. According to the thin-wall pipe structural behaviour, the following local stability constraints are proposed [16].

The stress constraint for against of Euler-buckling or peripheral shell-like buckling is given in terms of the thickness ratio.

$$
\begin{gathered}
\sigma_{e}^{E}=\frac{\pi E}{4 L^{2}} \cdot \frac{0.5-\alpha+\alpha^{2}}{\alpha(1-\alpha)} \cdot G_{e}, \\
\sigma_{e}^{B}=K E \alpha
\end{gathered}
$$

where $\alpha=T / D$ is the ratio of the wall-thickness and diameter of the applied $G_{e}$ group elements. In the present study, since continuous design variables are considered we applied tubular cross sections with given $\alpha=0.5$ thickness ratio.

In this paper, four different optimization problems has been solved, namely where

1 only stress constraints,

2 stress and displacement constraints,

3 stress and stability constraints, and

4 all of the constraints are considered.

The number of generations and the number of population size were 100 - 100 for each case detailed above.

The shape of the best solution to all cases (i-iv) is demonstrated on Fig. 36, where the shifted coordinates are signed in bracket.

The obtained best and worst values, the mean and the standard deviation of the statistical analysis of the 30 independent runs for all optimization problems are presented in Tab. 3

The cross-sectional areas of the best results selected from the 30 independent runs are presented in Tab. 4. Finally, in Tab. 5 the joint positions and weight of the previously discussed optimal results are compared with the published results using the same material properties.

Unfortunately, only the optimal weight and related optimal solution of geometrical configurations are presented in Pedersen [6] for each case. While in first case when only stress constraints are considered our results exhibit a good accordance with Pedersen's results, but in any other case the difference is considerable which might be arisen probably because of the nonlinear structural model applied in this study and the related stability constraints. 


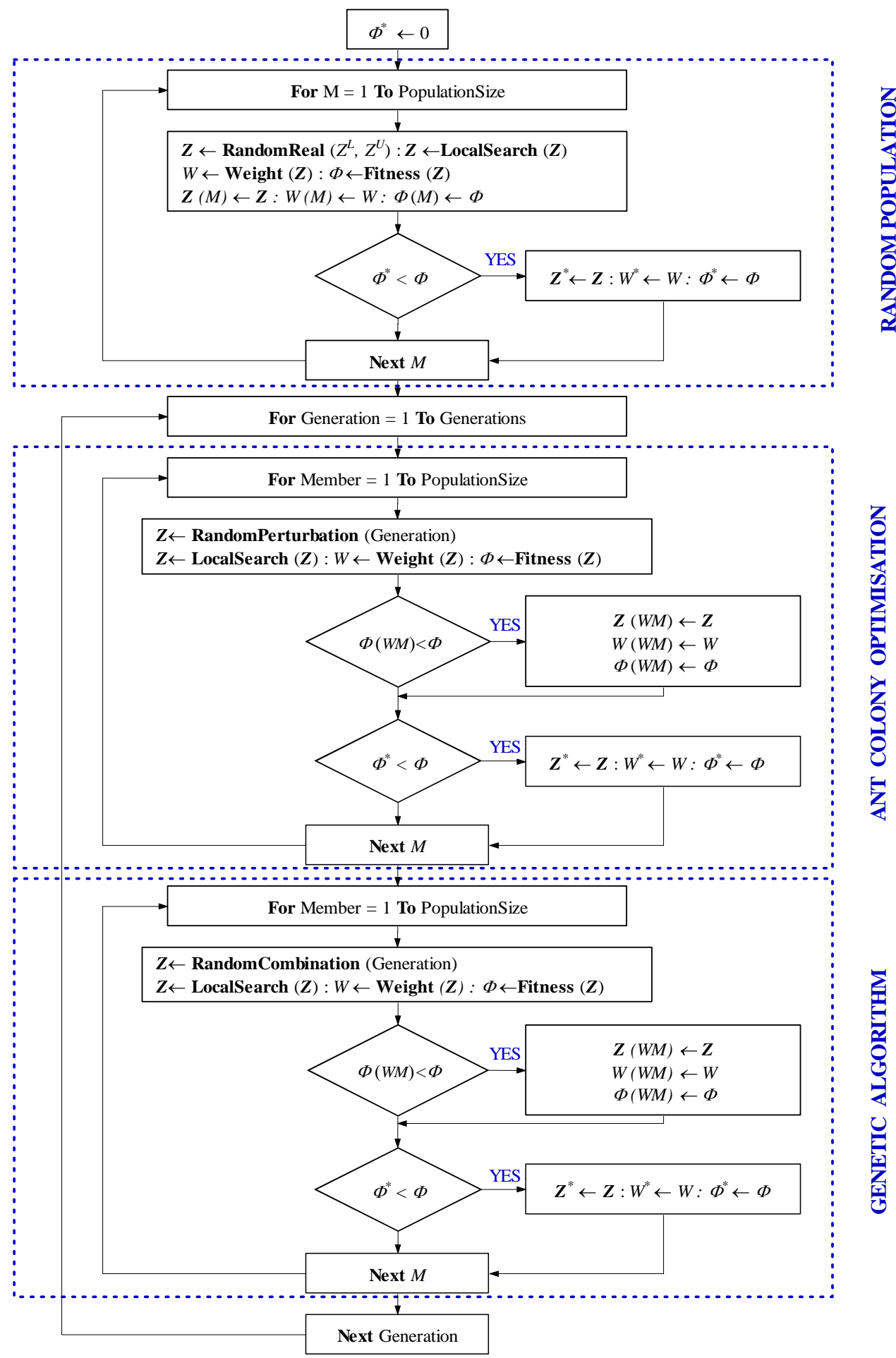

Fig. 1. The flowchart of the hybrid metaheuristic method

Tab. 1. The grouped cross sectional design variables

\begin{tabular}{cccccccccccccc}
\hline Group variables & $G_{1}$ & $G_{2}$ & $G_{3}$ & $G_{4}$ & $G_{5}$ & $G_{6}$ & $G_{7}$ & $G_{8}$ & $G_{9}$ & $G_{10}$ & $G_{11}$ & $G_{12}$ & $G_{13}$ \\
\hline Nodes & $1-7$ & $2-7$ & $1-6$ & $2-6$ & $3-6$ & $2-5$ & $3-5$ & $1-2$ & $2-3$ & $6-7$ & $5-6$ & $4-5$ & $3-4$ \\
\hline
\end{tabular}

The results revile the fact, that the displacement constraints, according to the moving load, significantly increase the complexity of the design space. Therefore, for a fixed searching pa- rameter set \{Generations, PopulationSize $\}$ the variability of the final results will be higher, which is well demonstrated by the range or the standard deviation of the "best" solutions in Tab. 3 . 
Tab. 2. Data for continuous sizing-shaping optimization of the Pedersen's truss-bridge

\begin{tabular}{|c|c|}
\hline \multicolumn{2}{|l|}{ Design variables: } \\
\hline Cross-sectional variables & $G_{i} \in[4,100]\left(\mathrm{cm}^{2}\right) ; \quad e \in\{1,2, \ldots, 13\}$ \\
\hline Geometry variables & $X_{5} ; \quad Y_{5} ; \quad X_{6} ; \quad Y_{6} ; \quad Y_{7}$ \\
\hline Stress constraints & $\sigma^{U}=130 \mathrm{MPa} ; \quad e \in\{1,2, \ldots, 13\}-\sigma_{e}^{L}=\max \left\{\sigma^{L}, \sigma_{e}^{E}, \sigma_{e}^{B}\right\} ; \sigma^{L}=-104 \mathrm{MPa} ; \quad e \in\{1,2, \ldots, 13\}$ \\
\hline Side constraints for geometry variables & $-250 \mathrm{~cm} \leq X_{5}, X_{6} \leq+250 \mathrm{~cm} ;-200 \mathrm{~cm} \leq Y_{3}, Y_{5}, Y_{7} \leq 300 q, \mathrm{~cm}$ \\
\hline $\begin{array}{l}\text { Displacement constraints of nodes } 1,2,3,8,9 \text {. } \\
\text { Load cases: }\end{array}$ & $u_{k}^{U}= \pm 1 \mathrm{~cm} ; \quad k \in\{1,2, \ldots, 5\}$ for vertical displacements \\
\hline Nodal points & In direction of coordinate y \\
\hline $1,2,3,8,9$ & $-300 \mathrm{kN}$ \\
\hline Material properties: & \\
\hline Modulus of elasticity & $E=210000 \mathrm{MPa}$ \\
\hline Density of the material & $\rho=7850 \mathrm{~kg} / \mathrm{m}^{3}$ \\
\hline
\end{tabular}

Tab. 3. Statistical analysis of 30 independent runs for truss-bridge optimization

\begin{tabular}{ccccc}
\hline Optimization cases & Only stress constraints & Displacement and stress constraints & Stress and stability constraints & All of the constraints \\
\hline Best weight $[\mathrm{kg}]$ & 1654.037 & 2794.259 & 1966.205 & 2866.116 \\
Worst weight $[\mathrm{kg}]$ & 1656.492 & 2819.659 & 1971.589 & 2934.523 \\
Mean $[\mathrm{kg}]$ & 1655.960 & 2803.280 & 1969.203 & 2894.924 \\
Standard deviation & 0.298 & 6.192 & 1.437 & 14.450 \\
\hline
\end{tabular}

Tab. 4. Optimal design of present study for shaping-sizing problems with consideration of design different constraints

\begin{tabular}{|c|c|c|c|c|c|}
\hline \multicolumn{2}{|c|}{ Geometry design variables } & \multirow{2}{*}{$\begin{array}{c}\text { Stress constraints } \\
11.1912\end{array}$} & \multirow{2}{*}{$\frac{\text { Displacement and stress constraints }}{15.5424}$} & \multirow{2}{*}{$\frac{\text { Buckling and stress constraints }}{10.306}$} & \multirow{2}{*}{$\frac{\text { All constrains }}{18.0787}$} \\
\hline$G_{1}$ & {$\left[\mathrm{~cm}^{2}\right]$} & & & & \\
\hline$G_{2}$ & $\left.\mathrm{~cm}^{2}\right]$ & 10.4305 & 18.6539 & 15.985 & 18.8412 \\
\hline$G_{3}$ & $\mathrm{~cm}^{2}$ & 8.1318 & 14.845 & 13.3364 & 17.1237 \\
\hline$G_{4}$ & $\mathrm{~cm}^{2}$ & 11.602 & 13.7711 & 11.757 & 16.8234 \\
\hline$G_{5}$ & $\left.\mathrm{~cm}^{2}\right]$ & 11.9618 & 11.4095 & 19.292 & 15.2628 \\
\hline$G_{6}$ & $\left.\mathrm{~cm}^{2}\right]$ & 5.4509 & 9.9113 & 6.7076 & 13.0312 \\
\hline$G_{7}$ & $\left.\mathrm{~cm}^{2}\right]$ & 13.6481 & 16.0756 & 16.3913 & 13.8369 \\
\hline$G_{8}$ & $\mathrm{~cm}^{2}$ & 4.0217 & 7.063 & 6.0943 & 7.3118 \\
\hline$G_{9}$ & $\mathrm{~cm}^{2}$ & 4.7628 & 10.0208 & 9.3416 & 9.4210 \\
\hline$G_{10}$ & $\left.\mathrm{~cm}^{2}\right]$ & 26.8054 & 48.2696 & 34.6539 & 50.4185 \\
\hline$G_{11}$ & {$\left[\mathrm{~cm}^{2}\right]$} & 24.3453 & 43.8197 & 27.3987 & 43.7335 \\
\hline$G_{12}$ & $\mathrm{~cm}^{2}$ & 29.8348 & 50.4848 & 31.2037 & 46.2772 \\
\hline $\mathrm{G}_{13}$ & $\left.\mathrm{~cm}^{2}\right]$ & 9.2574 & 10.4119 & 14.748 & 13.4586 \\
\hline
\end{tabular}

Tab. 5. Compared results for shaping-sizing optimization of the Pedersen's truss bridge

\begin{tabular}{|c|c|c|c|c|c|c|c|c|}
\hline \multirow[t]{2}{*}{ variables } & \multicolumn{2}{|c|}{ Stress constraints } & \multicolumn{2}{|c|}{ Displacement and stress constraints } & \multicolumn{2}{|c|}{ Buckling and stress constraints } & \multicolumn{2}{|c|}{ All constrains } \\
\hline & Pedersen [6] & Present study & Pedersen [6] & Present study & Pedersen [6] & Present study & Pedersen [6] & Present study \\
\hline$X_{5} \quad[\mathrm{~cm}]$ & -1153.00 & -1178.0864 & -1065.00 & -1059.8235 & -1138 & -1162.1736 & -1081 & -1072.8051 \\
\hline$X_{6} \quad[\mathrm{~cm}]$ & -633.00 & -621.8893 & -553.00 & -554.2587 & -629 & -588.3484 & -578 & -596.2107 \\
\hline$Y_{5} \quad[\mathrm{~cm}]$ & 437.00 & 433.5992 & 505.00 & 517.3998 & 266 & 404.4884 & 437 & 485.9064 \\
\hline$[\mathrm{cm}]$ & 672.00 & 665.3387 & 780.00 & 792.4968 & 485 & 525.5709 & 653 & 723.4665 \\
\hline$Y_{7} \quad[\mathrm{~cm}]$ & 753.00 & 746.4115 & 864.00 & 889.7917 & 500 & 575.6562 & 739 & 840.7323 \\
\hline$W \quad[k g]$ & 1656.00 & 1654.037 & 2911.00 & 2794.259 & 2905 & 1966.205 & 3315 & 2866.116 \\
\hline
\end{tabular}




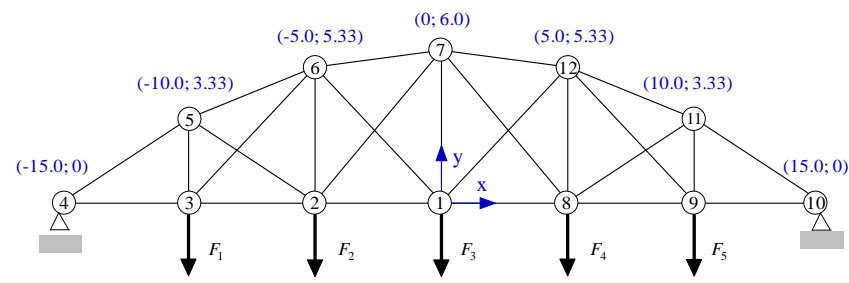

Fig. 2. The initial shape of the optimization problem

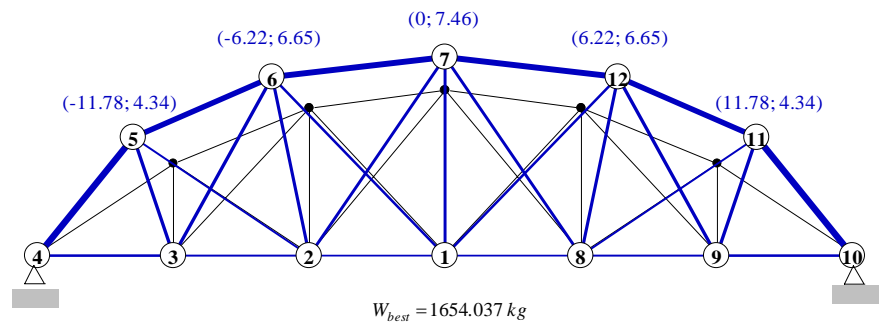

Fig. 3. Optimal design of only stress constrained optimization problem

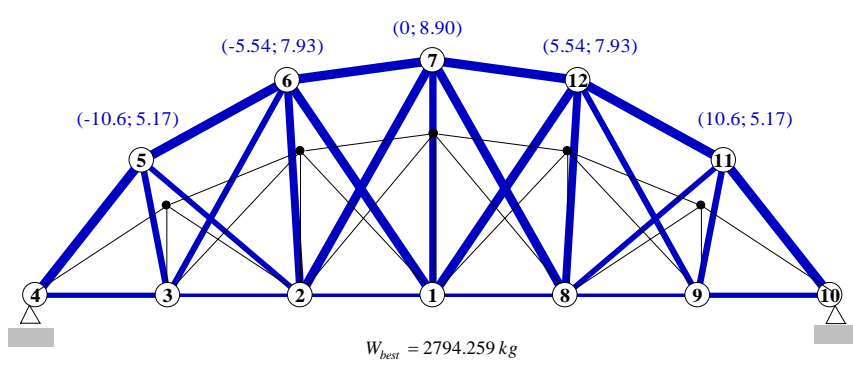

Fig. 4. Optimal design of stress and displacement constrained optimization problem

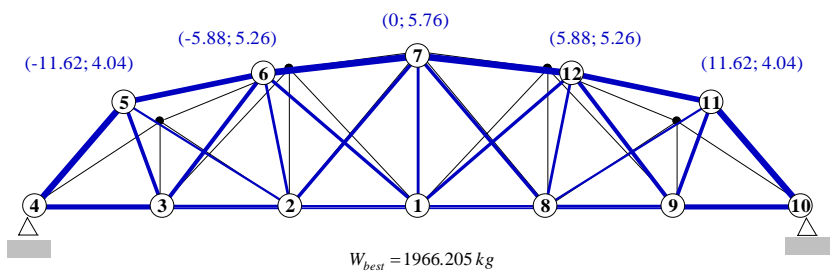

Fig. 5. Optimal design of buckling and stress constrained optimization problem

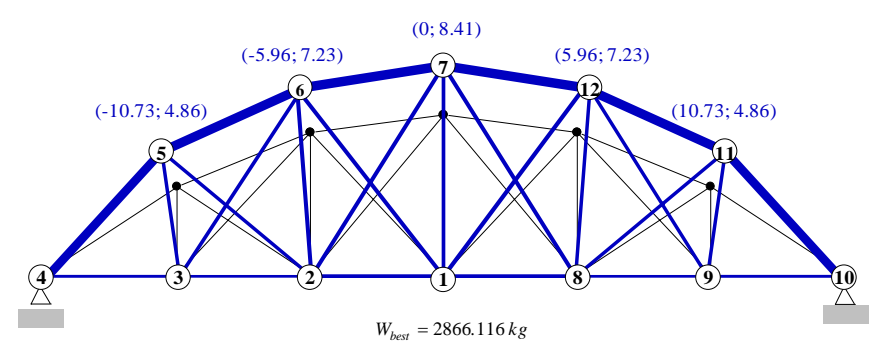

Fig. 6. Optimal design where all of the constraints are considered

\section{Conclusions}

In this paper, a hybrid metaheuristic method has been applied for multiple constrained truss optimization problems with continuous design variables. The proposed method combines ant colony optimization (ACO), genetic algorithm (GA), and local search strategy (LS) which seems an efficient mixture to solve simultaneous sizing-shaping truss optimization problems. The local search algorithm based on the local linearization has provided accurate results. Through a benchmark problem, which exhibits a large variety of the solutions, can be seen that the proposed hybrid algorithm seems very efficient and produces competitive results.

\section{References}

1 Kirsch U, Optimum Structural Design, McGraw-Hill, 1981.

2 Haftka R T, Gürdal Z, Kamat M P, Elements of Structural Optimization, Kluwer Academic Publisher, 1990.

3 Dobbs M W, Felton L P, Optimization of truss geometry, ASCE J Struct Div 95 (1969), 2105-2118.

4 Pedersen P, Optimal joint positions for space trusses, ASCE J Struct Div 99 (1973), 2459-2476.

5 , On the minimum mass layout of trusses, NATO Research and Technology Organization, AGARD Conference Proceedings No. 36 (AGARDCP36-70), 1970, pp. 11.1-11.17.

6 , On the optimal layout of multi-purpose trusses, Comput Struct 2 (1972), 695-712.

7 Vanderplaats G, Moses F, Automated design of trusses for optimum geometry, ASCE J Struct Div 98 (1972), 671-690.

8 Imai K, Configuration optimization of trusses by the multiplier method (Rep. No. UCLA-ENG-7842), PhD thesis, University of California, Los Angeles, CA, USA, 1978

9 Csébfalvi A, An ANGEL Method for Discrete Optimization Problems, Periodica Polytechnica Civ. Eng. 51 (2007), no. 2, 37-46, DOI 10.3311/pp.ci. 2007-2.06.

$10 \ldots$, An ANGEL Heuristic Method for Combined Shaping and Sizing Optimization of Bridges, Sixth International Conference on Engineering Computational Technology (Papadrakakis M and Topping, ed.), Civil-Comp Press, Stirlingshire, UK, 2008, pp. 1-13, DOI 10.4203/ccp.89.83, (to appear in print).

11 _ A Hybrid Method for Mixed Shaping-Sizing Optimization Problem, First International Conference on Soft Computing Technology in Civil, Structural and Environmental Engineering (Topping B H V and Tsompanakis, ed.), Civil-Comp Press, Stirlingshire, UK, 2009, pp. 1-19, DOI 10.4203/ccp.92.5, (to appear in print).

12 , A hybrid meta-heuristic method for continuous engineering optimization, Periodica Polytechnica Civ. Eng. 53 (2009), no. 2, 93-100, DOI 10.3311/pp.ci. 2009-2.05.

13 _ Nonlinear path-following method for computing equilibrium curve of structures, Annals of Operation Research 81 (1998), 15-23, DOI 10.1023/A:1018944804979.

14 Deb K, An efficient constraint handling method for genetic algorithm, Comp. Methods Appl. Mech. Engrg 186 (2000), 311-338.

15 Kaveh A, Talatahari S, Particle swarm optimizer, ant colony strategy and harmony search sheme hybridized for optimization of truss structures, Comput Struct 87 (2009), 267-283.

16 Rasmussen Kim JR, Recent research on stainless steel tubular structures, Journal of Constructional Steel Research 54 (2000), 75-88. 